\title{
Fundamental study of seismic emission tomography in terms of fluid pressure fluctuations
}

\author{
Akitomo WATANABE ${ }^{1}$, Hitoshi MIKADA ${ }^{1}$ and Junichi TAKEKAWA ${ }^{1}$ \\ ${ }^{1}$ Dept. of Civil and Earth Res. Eng., Kyoto University
}

\begin{abstract}
Recent years, seismic emission tomography which utilizes seismic oscillation due to fluid flow inside fractures has drawn more attention. However, the relationship between observed seismic data and fluid behavior in a reservoir has not been revealed yet. In the present study, we conduct numerical experiments for understanding the mechanism of the induced microseismic emission in order to extract more information about fluid behavior from observed seismic data. We simulate fluid flow in a fracture using the lattice Boltzmann method (LBM). We adopt two numerical models, i) parallel plate model, and ii) pore throat model. We calculate stress changes at the fracture wall induced by unsteady flow and multi-phase flow fields. The unsteady flow is generated by cyclic pressure change at the inflow boundary. In this case, inner portion of the fracture is filled only water or oil. In the multi-phase flow, we consider migration of oil droplet in a fracture with a throat filled by water. In the parallel plate model, larger shear stress change can be observed in the case of oil. This stems from more rapid change in fluid velocity close to the fracture wall due to the high viscosity of oil. In the case of the multi-phase flow in the pore throat model, about 8 $\mathrm{Pa}$ of shear stress and $28 \mathrm{~Pa}$ of normal stress are observed at the fracture wall when an oil droplet whose diameter is $1 \mathrm{~mm}$ passes through the pore throat. We estimate where fluid flowing using seismic wave from that stress changes. Our results show that the induced microseismic emission by fluid flow is strongly dependent on the fluid viscosity, geometry of fracture network, etc., which influences the pattern and the flux of the flow.
\end{abstract}

\section{INTRODUCTION}

Hydraulic fracturing has been used to develop unconventional reservoirs like shale oil, shale gas and geothermal reservoirs. Although the evaluation of the extent of reservoirs and complicated fracture network is required, it is difficult to estimate the above information because of the lack of the mechanism of hydraulic fracturing. So it is important to establish a monitoring technique for understanding fracture behavior and fluid flow in the sub-surface. Recent years, seismic emission tomography, which utilizes seismic signals induced by fluid flow inside reservoirs, has drawn attention to visualize the subsurface ${ }^{(12}$. In study of passive seismic emission tomography, there are many studies focus on acoustic emission of hydraulic fracturing and boring well ${ }^{3}$. However, the relationship between observed seismic data and fluid behavior in the subsurface has not been well understood yet.

In the present study, we conduct numerical experiments to understand the mechanism of seismic emission. We set a single-phase flow model and a multi-phase flow model to investigate the effect of pressure oscillation and inhomogeneous flow on emitted seismic waves. Received waveforms at surface receivers are calculated by the
Green function based on induced stress changes at the fracture wall. We quantitatively evaluate the effectiveness of passive seismic emission tomography using our numerical results.

\section{METHOD}

In this paper, we use the lattice Boltzmann method (LBM) to simulate two-phase fluid flow. In the LBM, the two-dimensional nine-directional (D2Q9) lattice Bhatnager-Gross-Krook model (LBGK) is adopted. In this model, the directions of the discrete velocity are given as

$$
c_{i}=\left\{\begin{array}{c}
0 \text { for } i=1 \\
1 \text { for } i=2,3,4,5 \\
\sqrt{2} \text { for } i=6,7,8,9
\end{array}\right.
$$

The evolution equation of the particle velocity distribution function $f_{i}(x, t)$ leads

$$
f_{i}^{k}\left(x+c_{i} \Delta t, t+\Delta t\right)=f_{i}^{k}(x, t)+\omega_{i}^{k}
$$

where $\mathrm{k}=\mathrm{r}$ or $\mathrm{b}$ donates the color ('red' or 'blue') for multi-phase flow. 


$$
\begin{aligned}
& \omega_{i}^{k}(x, t)=\left(\omega_{i}^{k}(x, t)\right)^{B G K} \\
& +\left(\omega_{i}^{k}(x, t)\right)^{I F T}
\end{aligned}
$$

The calculation of $\omega_{i}^{k}(x, t)$ consists of two processes. The first represents relaxation to a local equilibrium state using, for simplicity, an LBGK operator:

$$
\begin{aligned}
\left(\omega_{i}^{k}(x, t)\right)^{B G K} & =-\frac{1}{\tau}\left(f_{i}^{k}(x, t)\right. \\
& \left.-f_{i}^{k, e q}(x, t)\right)
\end{aligned}
$$

where the relaxation time $\tau$ is a parameter which characterizes the constitutive behavior of the fluid at a macroscopic level. It is related to the macroscopic kinetic viscosity $v$ of the fluid as:

$$
v=\frac{c^{2}}{3}\left(\tau-\frac{1}{2}\right) \Delta t
$$

$f_{i}^{e q}(x, t)$ is the local equilibrium distribution function. In order to develop the correct Navier-Stokes equations, the local equilibrium distribution function is defined as:

$$
\begin{aligned}
& f_{i}^{k, e q}(x, t)=\rho_{k} {\left[D_{i}^{k}\right.} \\
&\left.+w_{i}\left\{3 c_{i} \cdot v-\frac{3}{2} v^{2}\right\}\right]
\end{aligned}
$$

where $w_{i}$ and $D_{i}$ represent the weight factors. $\rho$ is the density and $v$ is the flow velocity vector given as follows:

$$
\begin{aligned}
& w_{i}= \begin{cases}4 / 9 & \text { for } i=1 \\
1 / 9 & \text { for } i=2,3,4,5 \\
1 / 36 & \text { for } i=6,7,8,9\end{cases} \\
& \rho_{k}(x, t)=\sum_{i} f_{i}^{k}(x, t) \\
& v(x, t)=\frac{1}{\rho} \sum_{i} c_{i} \sum_{k} f_{i}^{k}(x, t) \\
& D_{i}^{k}=\left\{\begin{array}{cl}
\alpha_{k} & \text { for } i=1 \\
\left(1-\alpha_{k}\right) / 5 & \text { for } i=2,3,4,5 \\
\left(1-\alpha_{k}\right) / 20 & \text { for } i=6,7,8,9
\end{array}\right.
\end{aligned}
$$

where $\alpha_{k}$ is dependent on density ratio of two fluid.

$$
\gamma=\frac{\rho_{a}}{\rho_{b}}=\frac{\left(1-\alpha_{b}\right)}{\left(1-\alpha_{a}\right)}
$$

The second process is the two-phase component of the collision operator:

$$
\begin{aligned}
\left(\omega_{i}^{k}(x, t)\right)^{I F T}= & \frac{A}{2}|F|\left\{w_{i} \frac{\left(c_{i} \cdot F\right)^{2}}{|F|^{2}}\right. \\
& \left.-B_{i}\right\}
\end{aligned}
$$

where $B_{i}$ represent the weight factors. $A$ is a parameter of surface tension. $F(x, t)$ is color gradient which is given as follows:

$$
\begin{gathered}
B_{i}=\left\{\begin{array}{cc}
-4 / 27 & \text { for } i=1 \\
2 / 27 & \text { for } i=2,3,4,5 \\
5 / 108 & \text { for } i=6,7,8,9
\end{array}\right. \\
F(x, t)=\sum_{i} c_{i}\left\{\rho_{r}\left(x+c_{i}, t\right)\right. \\
\left.-\rho_{b}\left(x+c_{i}, t\right)\right\}
\end{gathered}
$$

Using this simulation scheme, we exploit two-phased fluid simulation. We check this scheme work good or not using Laplace's law. Laplace's law is given as follows:

$$
\mathrm{P}_{\text {in }}-\mathrm{P}_{\text {out }}=\frac{\sigma}{\mathrm{R}}
$$

where $\mathrm{R}$ is radius of droplet and $\sigma$ is boundary tension. We set oil in square shape, and execute simulation. We show the initial state and static state in Fig.1.

We execute simulation changing oil droplet size, we calculate $\mathrm{P}_{\text {in }}-\mathrm{P}_{\text {out }}(=\Delta \mathrm{P})$. We show relationship radius of droplet and boundary tension in Fig.2. It show inverse proportion between $\mathrm{R}$ and $\sigma$. This result agrees with Laplace's law, we can consider our simulation work accurately.

In this paper, we use Green function to calculate seismic waves which is caused by oil droplet through pore-throat. When external force is shown by $f(x, t)$, wave equation is given as:

$$
\frac{1}{\alpha^{2}} \frac{\partial^{2} \varphi}{\partial t^{2}}=\nabla^{2} \varphi(x, t)+f(x, t)
$$

Using Green function $G\left(x, t ; x_{0}, t_{0}\right)$, that wave equation's solution is given as:

$$
\begin{aligned}
& \varphi(x, t) \\
& =\int_{-\infty}^{+\infty} d t_{0} \int_{V} G\left(x, t ; x_{0}, t_{0}\right) f\left(x_{0}, t_{0}\right) d V_{0}
\end{aligned}
$$


If we calculate the Green function only once, arbitrary responses can be obtained by the superposition.
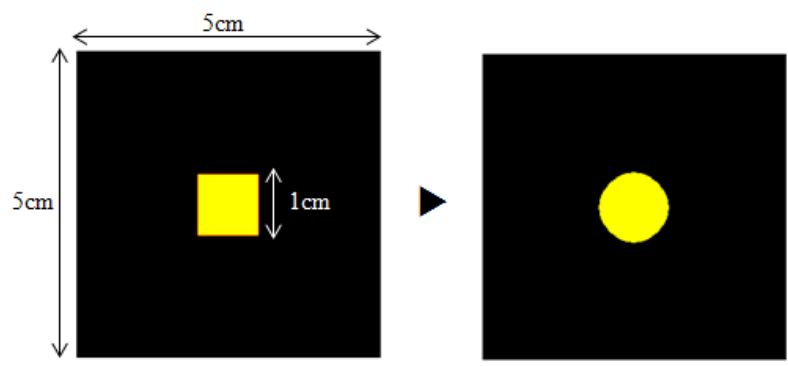

Fig.1 initial state (left figure) and static state (right figure) of oil droplet

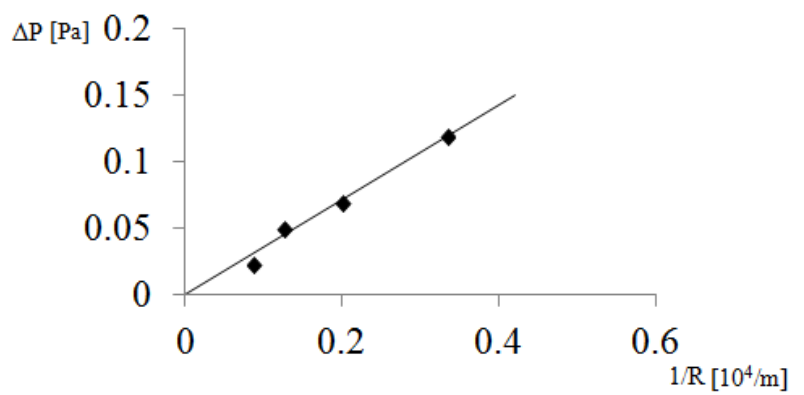

Fig.2 simulation result (dots) and theoretical value from Laplace's law (solid line)

\section{MODEL}

In this paper, we use two numerical models, i) periodic flow in a parallel plate (Fig.3), and ii) a pore throat model with two-phase (Fig.4). We calculate stress changes at the fracture wall induced by unsteady flow for i) and multi-phase flow for ii). The unsteady flow is generated by cyclic pressure change at the inflow boundary. In this case, we use single-phase flow. In the multi-phase flow, we consider transport of an oil droplet in a fracture with a throat filled by water. Physical and numerical parameters are shown in Table 1,2 and 3.

After those experiments, we simulate seismic wave propagation and calculate received waveforms at surface geophones using Green function.

Table.1: Common parameters of parallel plate model and pore-throat model

\begin{tabular}{|c|c|}
\hline Kinematic viscosity (oil) & $1 \times 10^{-6} \mathrm{~m}^{2} / \mathrm{s}$ \\
\hline Kinematic viscosity (water) & $1 \times 10^{-5} \mathrm{~m}^{2} / \mathrm{s}$ \\
\hline Density (oil) & $900 \mathrm{~kg} / \mathrm{m} 3$ \\
\hline Density (water) & $1000 \mathrm{~kg} / \mathrm{m} 3$ \\
\hline
\end{tabular}

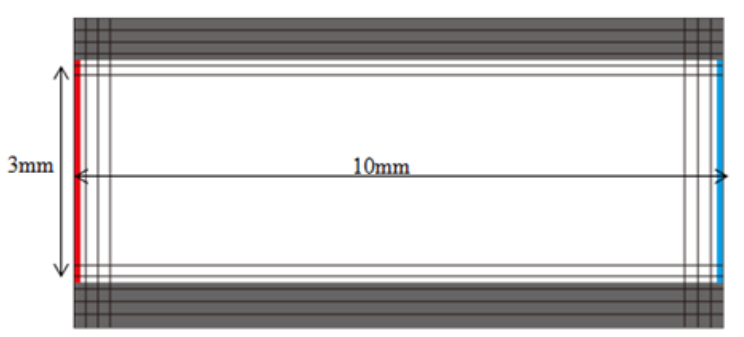

Fig.3: Parallel plate model

Table 2: Parallel plate model parameters

\begin{tabular}{|c|c|}
\hline$\Delta \mathrm{x}$ & $1 \times 10^{-4} \mathrm{~m}$ \\
\hline$\Delta \mathrm{t}$ & $1 \times 10^{-4} \mathrm{~s}$ \\
\hline $\begin{array}{c}\text { Maximum pressure } \\
\text { difference }\end{array}$ & $1000 \mathrm{~Pa}$ \\
\hline
\end{tabular}

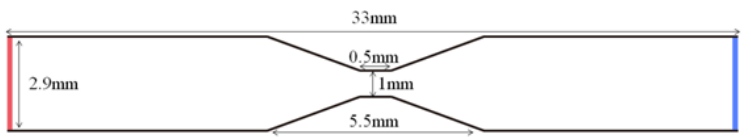

Fig.4: Pore-throat model

Table 3: Model ii parameter

\begin{tabular}{|c|c|}
\hline$\Delta \mathrm{x}$ & $5 / 6 \times 10^{-4} \mathrm{~m}$ \\
\hline$\Delta \mathrm{t}$ & $1 \times 10^{-4} \mathrm{~m}$ \\
\hline Pressure difference & $18.52 \mathrm{~Pa}$ \\
\hline
\end{tabular}

We set pore-throat at $(\mathrm{x}, \mathrm{y}, \mathrm{z})=(0,0,-1000)$, and its axis is parallel to $\mathrm{x}$-axis. We set geophones on $81 \mathrm{~m}$ lattice spacing at the surface as shown in Fig.5. On the assumption of homogeneous infinite medium, we use Green function solution to simulate seismic waves.

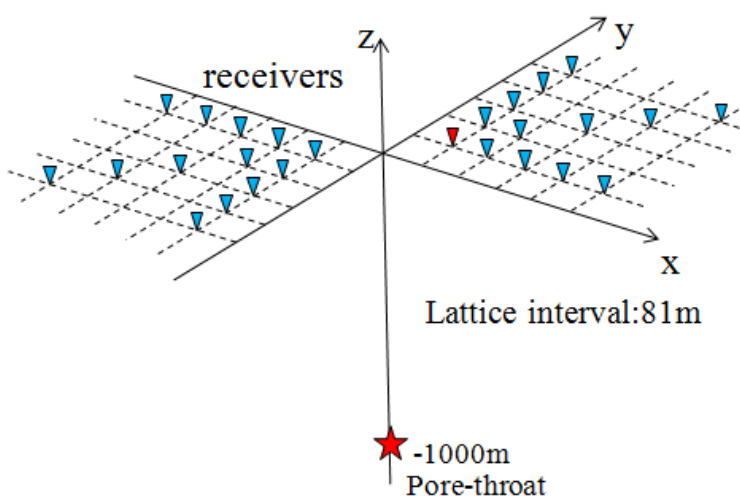

Fig.5: Observation model

\section{RESULT}

Fig.6 shows the time variation of the shear stress at the fracture wall in the parallel plate model. Larger shear stress change can be observed in the case of oil than water. This stems from more rapid change in fluid velocity close to the fracture wall due to the high viscosity of oil. This result indicates 
seismic wave with fluid flow will be changed what fluid exist in fracture and possibility that we can estimate kind of fluid in fracture with observing seismic waves.

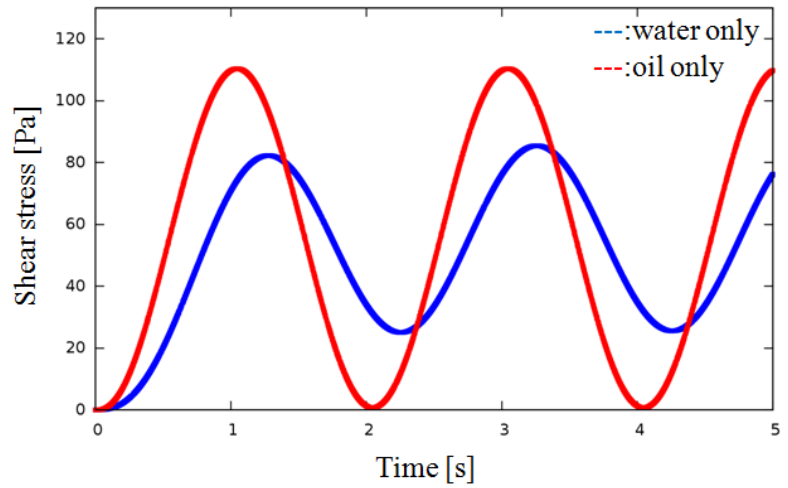

Fig.6: Shear stress in parallel plate model

Fig. 5 shows snapshots of the passage of the oil droplet in the pore-throat model. The migration of the droplet is driven by the pressure gradient. The droplet passes through the throat with deformation and frictioning. The droplet completely reaches the right side pore at $0.163 \mathrm{~s}$.
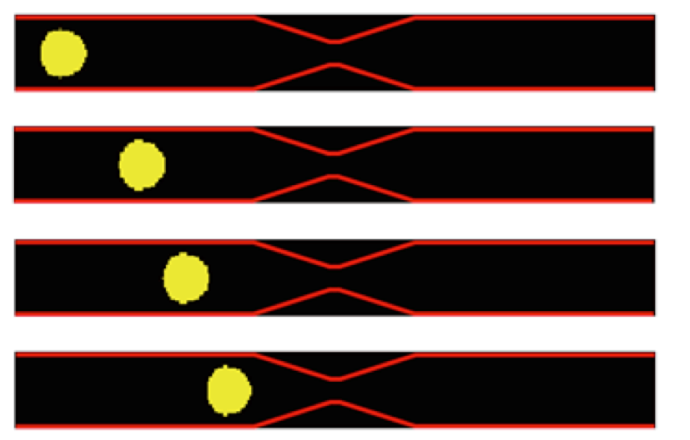

0.100
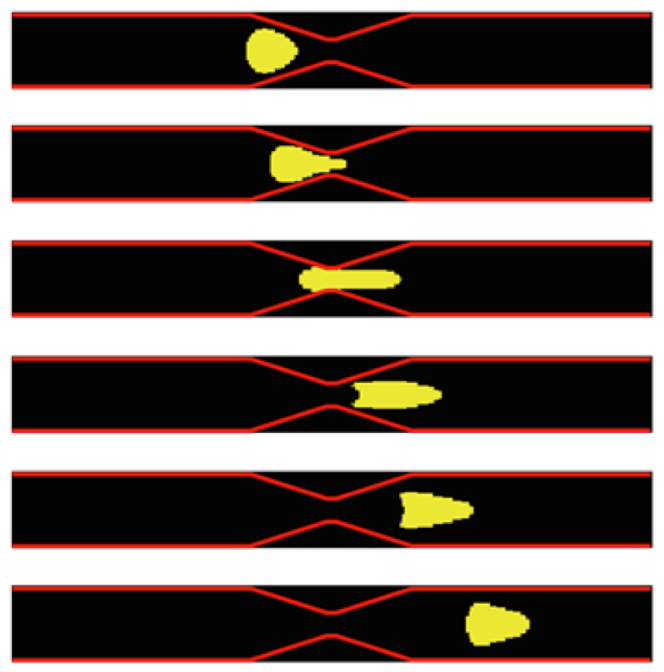

0.200

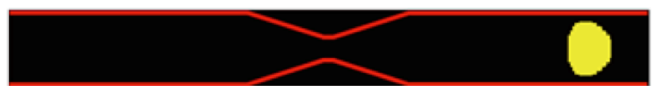

0.250

Fig.7: Time change of moving oil droplet
Fig.6 and Fig.7 shows the time variation of the normal and shear stresses at the fracture wall in the case of the multi-phase flow in the pore throat model. We also show the results of single-phase flow of water as examples. About $8 \mathrm{~Pa}$ and $28 \mathrm{~Pa}$ of shear and normal stress changes are observed at the fracture wall when an oil droplet passes through the pore throat, whose width is $1 \mathrm{~mm}$. Our results show that the induced microseismic emission by fluid flow is strongly dependent on the fluid viscosity, geometry of fracture network and so on.

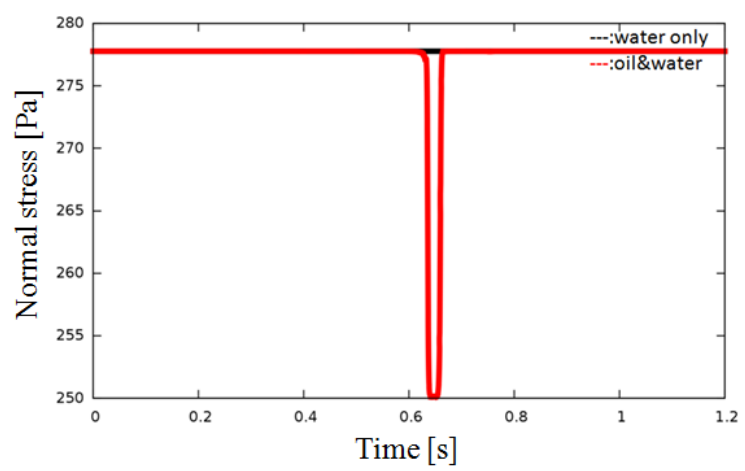

Fig.8: Normal stress in pore-throat model

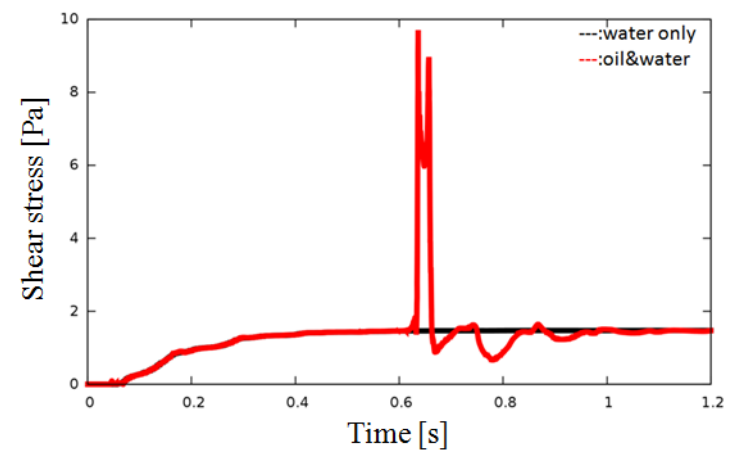

Fig.9: Shear stress in pore-throat model

Using the induced stress changes, we calculate received waveforms at surface geophones which set on $\quad(x, y, z)=(81,81,0)$. Pore-throat set on $(\mathrm{x}, \mathrm{y}, \mathrm{z})=(0,0,-1000)$. Fig. 8 shows an example of received waveforms. When one oil droplet passes through the pore throat, geophones detect seismic wave whose amplitude is of the order of 10-27 m. Although this amplitude cannot be detected in field observations, the superposition of waveforms induced by many droplets at many pore throats can amplify the signal from the reservoir. Therefore we calculate cross correlation and autocorrelation of those receivers to enhance the signal. We assume many pore-throats in the subsurface emit induced microseismic emission at random timing. By considering 16000 pore throats in the subsurface and taking correlation of received seismic waves for 20 days, the amplitude of passive seismic emission tomography can be enhanced at the same level as general acoustic emission due to fracturing. 


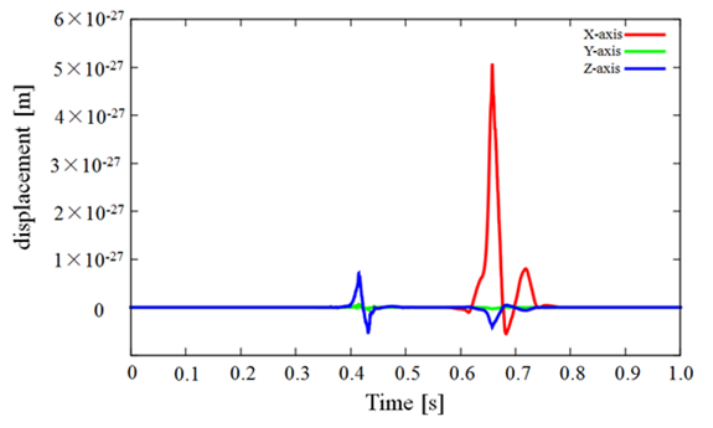

Fig.10: Received waveform

In Fig.11 we show example of received wave at geophone $\mathrm{A}$ and $\mathrm{B}$. and we show the auto correlation of received wave $\mathrm{A}$ and cross correlation of received wave A and B. Peak time difference of auto-correlation and cross correlation result from positional relation of receivers and oscillation point.

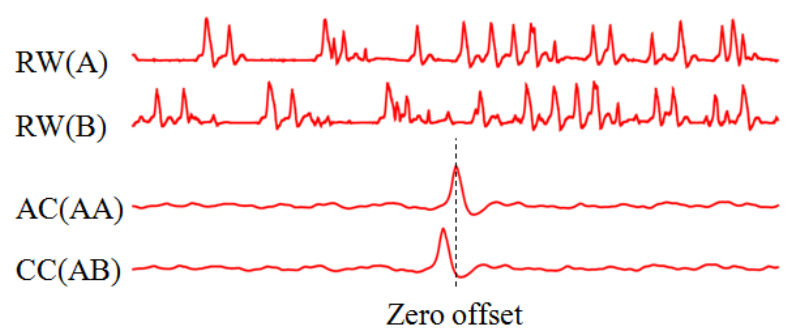

Fig.11 example of correlation

RW(A) :received wave of geophone A

AC(AA):auto correlation of RW(A)

$\mathrm{CC}(\mathrm{AB})$ :cross correlation of RW(A) and (B)

We take auto correlation at a red receiver in Fig.5 and cross correlation of a red receiver and blue receivers in Fig.5. Using auto correlation and cross correlation, we can estimate where fluid flow. We demonstrate the effectiveness of passive seismic emission tomography using an imaging of the location of the pore-throat. The imaged result is shown in Fig.12. The true oscillation point is

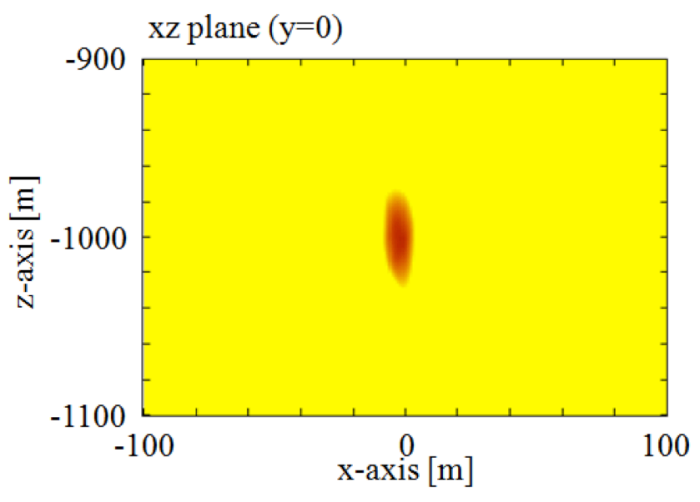

Fig.12: Estimated oscillation point.

True points is $(\mathrm{x}, \mathrm{y}, \mathrm{z})=(0,0,-1000)$

Estimated points is $(\mathrm{x}, \mathrm{y}, \mathrm{z})=(-2,1,-1002)$ $(\mathrm{x}, \mathrm{y}, \mathrm{z})=(0,0,-1000) . \quad$ On the other hand, our estimated oscillation point is $(\mathrm{x}, \mathrm{y}, \mathrm{z})=(-2,1,-1002)$. It suggests possibility that we can estimate where fluid flowing using correlation of received waveform.

\section{DISCUSSION AND CONCLUSION}

We calculate fluid flow and stress changes at the fracture wall using LBM. We then calculate seismic waves generated by the stress changes at the fracture wall using a Green function's solution. Our results show that pressure oscillation and inhomogeneous flow could be sources of induced microseismic emission. The result from the parallel plate model indicates that the emitted microseismic emission induced by stress change at the fracture wall would have information about the kind of fluid passing along solid-fluid interfaces.

We quantitatively evaluate the effectiveness of the passive seismic emission tomography using synthetic waveforms obtained by the Green function. By taking correlation of the received waveforms, seismic source region can be imaged for realistic observation terms. In our model, we assume 16000 pore-throats exist in the source region. However, there must be much more pore-throats in the real reservoirs ${ }^{(4}$. Our numerical simulations and quantitative analyses show that the passive seismic emission tomography can reveal not only the location of seismic source region but also the physical parameters of fluid.

\section{REFERENCES}

1) Erokhin, G. N., et al., 2014, Small microseismic surface acquisition system case study. 76th EAGE Conference and Exhibition Workshops. DOI: $10.3997 / 2214-4609.20140576$

2) Maxwell, Shawn C., and Urbancic, Theodore I., 2001, The role of passive microseismic monitoring in the instrumented oil field. The Leading Edge 20 (6), 636-639.

3) Eisner, Leo, et al., 2010, Comparison of surface and borehole locations of induced seismicity. Geophysical Prospecting 58 (5), 809-820.

4) Madonna, C., Quintal, B., Frehner, M., Almqvist, B. S. G., Tisato, N., Pistone, M., Marone, F., and Saenger, E. H., 2013, Synchrotron-based $\mathrm{X}$-ray tomographic microscopy for rock physics investigations, Geophysics, 78 (1), D53-D64. 